\title{
Segmentação de Lesões de Pele Utilizando Algoritmos de Aprendizagem Profunda
}

\author{
Lucas B. M. de Souza ${ }^{1}$ Samuel Pedro B. D. Lélis ${ }^{1}$, Romuere R. V. Silva ${ }^{1}$ \\ ${ }^{1}$ Sistemas de Informação, Universidade Federal do Piauí (UFPI) \\ Picos - PI - Brazil \\ \{lucasbezerra527, samuellelis88\}@gmail.com, romuere@ufpi.edu.br
}

\begin{abstract}
Among the skin cancer types, melanoma is the most serious and frequent in Brazil, besides, it has a growing incidence all over the world, showing the importance of automated systems for detection of this kind of cancer in medical images for a fast diagnosis. One of the steps is the segmentation of the region with the lesion. In this paper, we used a series of post-processing techniques (Otsu's method, region filling and small object removal) improving the results when compared to the proccess using a deep learning-based algorithm with the framework $U$-Net. We achieved results in the $P H^{2}$ base of 0.9482 Dice value, and 0.7729 for the ISIC 2018 base.
\end{abstract}

Resumo. Dentre os cânceres de pele, o melanoma é o mais grave e mais frequente no Brasil. Além disso, sua incidência está aumentando cada vez mais pelo mundo, assim mostrando a importância dos sistemas de detecção do câncer através de imagens médicas para a obtenção de um diagnóstico mais rápido. Uma das etapas é a segmentação, que trata do isolamento da região lesionada. Nesta pesquisa realizou-se a aplicação das técnicas de pós-processamento limiar de Otsu, fechamento de regiões e remoção de pequenas regiões para meIhorar o resultado com algoritmos baseados em aprendizagem profunda U-Net. Com a utilização das bases $\mathrm{PH}^{2}$ e ISIC 2018, foram obtidos 0,9482 e 0,7729 de valores de Dice, respectivamente.

\section{Introdução}

Segundo o Instituto Nacional de Câncer (INCA), o câncer de pele é o mais frequente no Brasil, detendo cerca de $30 \%$ de todos os tumores malignos registrados no país, sendo $3 \%$ representado pelo melanoma. A estimativa para novos casos atualizada em 2020 é de 8.450, sendo 4.200 homens e 4.250 mulheres [Inca b]. O número de mortes em decorrência do câncer de pele do tipo melanoma em 2018 no Brasil foi de 1.790, sendo 1.038 homens e 752 mulheres [Inca a].

Dentre os tipos de câncer de pele, o mais grave é o melanoma, conhecido como melanoma cutâneo, que se origina nos melanócitos (células produtoras de melanina). Conforme [Zhang et al. 2020], este câncer é o motivo da maioria das mortes por câncer de pele, sendo fatores genéticos e radiação ultravioleta as principais causas envolvidas. Os principais sintomas são manchas pruriginosas, sinais ou pintas que mudam de tamanho, forma ou cor e feridas que não cicatrizam em 4 semanas [Ministério da Saúde ].

Quando o melanoma é detectado nos estágios iniciais há mais chances de cura e de sobrevivência dos pacientes. Isso porquê é um câncer perigoso que pode provocar metástase (disseminação do câncer para outros órgãos). Assim, cada vez mais 
a computação vem trabalhando junto à saúde, sendo comum o termo Computer-Aided Detection (CAD) em trabalhos científicos que tratam de computação aplicada à saúde. Esse tipo de sistema pode ser desenvolvido para detectar o melanoma e agilizar o diagnóstico, com objetivo de obter laudos automáticos, com menor custo e maior eficiência [Araújo et al. 2019].

Em trabalhos como Nazi and Abir 2020, são propostos algoritmos que conseguem classificar a lesão em benigna ou maligna. Com o objetivo de melhorar a classificação foi feita a segmentação da região de interesse (ROI), ou seja, foi removida da imagem a porção que não constitui lesão. Cicerone and Camp 2019 afirma que abordagens de CAD podem ajudar o patologista com o fornecimento de métricas de imagem para calcular vários diagnósticos, sendo uma delas a identificação de potenciais ROIs, pois proporciona uma economia de tempo significativa para o fornecimento do diagnóstico.

Dessa forma, o objetivo deste trabalho é utilizar uma rede convolucional baseada na arquitetura U-Net [Ronneberger et al. 2015] para segmentação de imagens de lesões de pele e aliar o resultado a técnicas de pós-processamento. A fim de mostrar a efetividade do método, este foi testado em duas base de dados, sendo elas $\mathrm{PH}^{2}$ [Mendonça et al. 2013] e ISIC 2018 [Codella et al. 2019], além de comparar com métodos do estado da arte.

\section{Trabalhos Relacionados}

No trabalho [Al-masni et al. 2018], foi proposta uma nova metodologia de segmentação com base em redes neurais convolucionais de resolução total [Long et al. 2015]. A rede proposta não necessita de pré ou pós-processamento para a remoção de artefatos ou do aprimoramento da lesão segmentada. Uma das bases de dados utilizadas para avaliar o método foi a $\mathrm{PH}^{2}$, na qual obteve-se 91,38\% de coeficiente Dice.

Visando a resolução de problemas de desequilíbrio de dados e de desempenho no treinamento da U-Net tradicional, o trabalho proposto por Abraham and Khan 2018 combinou a U-Net com uma nova variante da função de perda focal [Lin et al. 2020], obtendo uma rede mais adequada à segmentação de pequenas lesões. Foi usada a base de imagens ISIC 2018, na qual a segmentação atingiu 0,856 para o Dice.

[Kim et al. 2017] obtiveram uma média de Dice na base de imagens $\mathrm{PH}^{2}$ de 0,940 . Para segmentação das imagens foi desenvolvida uma rede de aprendizado profundo iterativa de codificador-decodificador, que consiste em camadas de convoluções para codificador e camadas de deconvolução para decodificador. Esse método propõe a segmentação de várias imagens médicas, sendo testado na base $\mathrm{PH}^{2}$ e na base de imagens de retina DRIVE [Staal et al. 2004].

Em [Nazi and Abir 2020], foi utilizada uma rede convolucional U-Net para segmentar imagens, obtendo uma média 0,87 com o Dice. A base de dados utilizada foi a ISIC 2018. Devido ao número de imagens, foram realizados os aumentos de dados: rotação aleatória, inversão aleatória, zoom aleatório, distorção gaussiana, brilho aleatório, cor aleatória, contraste aleatório, distorção elástica aleatória e equalização do histograma para que se tornasse possível treinar e testar melhor o modelo. Foi utilizada também a base $\mathrm{PH}^{2}$ atingindo 0,93 de Dice.

A rede neural convolucional U-Net utilizada nesse trabalho é a mesma proposta por [Ronneberger et al. 2015], que ganhou o prêmio ISBI 2015 [IEEE ]. Foi mostrado 
que essa rede pode ser treinada de ponta a ponta a partir de apenas 30 imagens, sendo uma das principais vantagens a possibilidade de utilizar bases com poucas imagens para a aplicação desse modelo no meio acadêmico.

O nosso trabalho consiste na utilização a U-Net conforme proposto [Ronneberger et al. 2015], aliado a técnicas de pós-processamento e alterações nos parâmetros de otimização, quantidade de épocas e taxa de aprendizagem para a proposição de um método possivelmente mais eficiente na segmentação das imagens de câncer de pele, visando obter resultados melhores que os trabalhos do estado da arte.

\section{Materiais e Métodos}

O método proposto tem o objetivo de melhorar os resultados de uma segmentação de melanoma providos através de uma rede neural convolucional U-Net. A imagem a seguir constitui o detalhamento do método proposto.

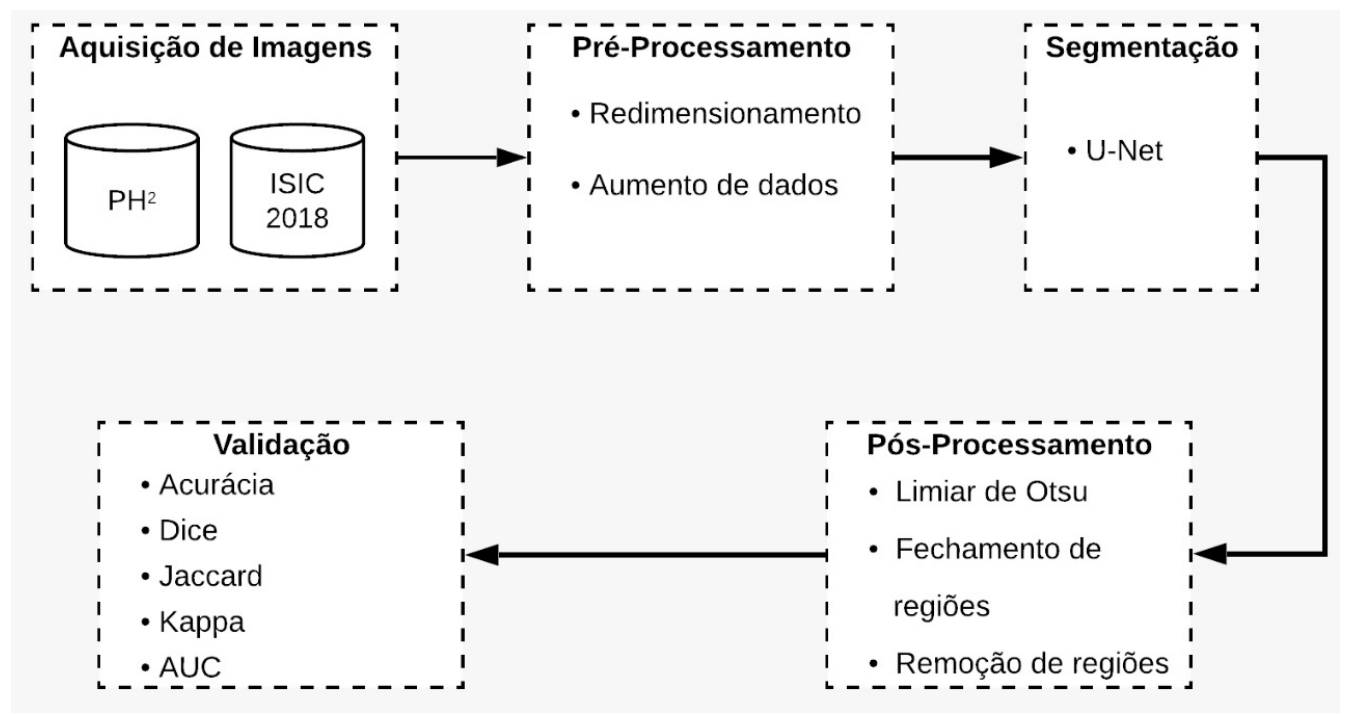

Figura 1. Fluxograma de etapas da metodologia.

\subsection{Aquisição de Imagens}

No processo de aquisição, são reunidas as imagens que contém as lesões cutâneas e as segmentadas manualmente por um médico (máscaras). Foram utilizadas duas bases, a $\mathrm{PH}^{2}$ e a ISIC 2018.

De acordo com [Universidade do Porto ] e [Mendonça et al. 2013], o conjunto de dados $\mathrm{PH}^{2}$ foi desenvolvido para fins de pesquisa e benchmarking, a fim de facilitar estudos comparativos sobre algoritmos de segmentação e classificação de imagens dermatoscópicas. Tal base contém um total de 200 imagens, sendo 160 não melanomas e 40 melanomas. Na Figura 2, em (a) tem-se um exemplo de uma imagem de lesão não melanoma e em (b) sua máscara, em (c) um exemplo de melanoma e (d) sua máscara. 


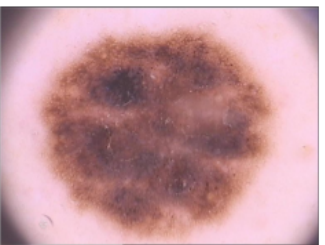

(a)

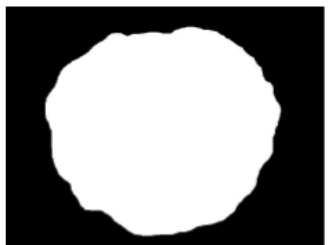

(b)

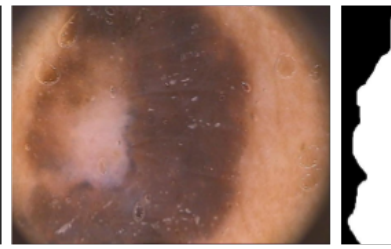

(c)

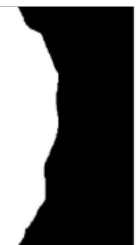

(d)

Figura 2. Exemplo lesão e máscara $\mathbf{P H}^{2}$, sendo (a) não melanoma e (b) sua máscara, (c) melanoma e (d) máscara.

A base de imagens ISIC 2018 foi criada pela a International Skin Imaging Collaboration (ISIC) [ISIC ], e constitui um esforço internacional para a melhora no diagnóstico de melanoma, patrocinado pela Sociedade Internacional de Imagem Digital da Pele (ISDIS). A base foi disponibilizada em uma competição onde vários pesquisadores tentaram desenvolver o melhor método dentre as categorias estabelecida na competição [International Skin Imaging Collaboration ]. A base contém 2594 imagens, sendo 2075 não melanomas e 519 melanomas. Na Figura 3, em (a) temos um exemplo de uma imagem de lesão não melanoma e em (b) sua máscara, em (c) um exemplo de melanoma e (d) sua máscara.

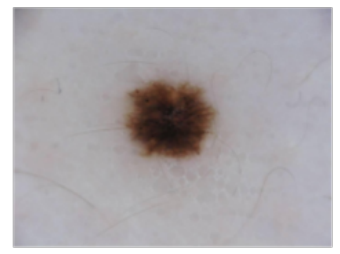

(a)

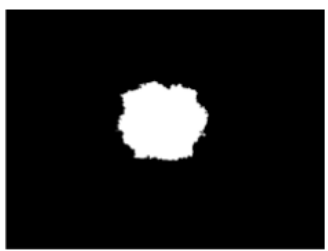

(b)

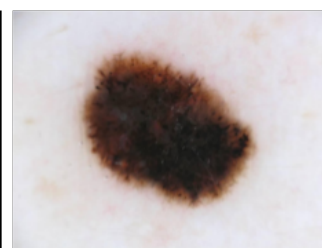

(c)

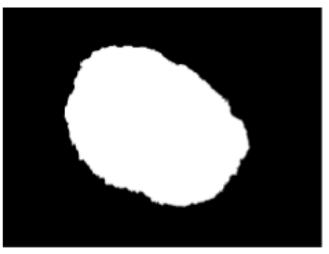

(d)

Figura 3. Exemplo lesão e máscara ISIC 2018, sendo (a) não melanoma e (b) sua máscara, (c) melanoma e (d) máscara.

\subsection{Pré-processamento}

Nessa etapa foram preparadas as imagens a serem utilizadas nas etapas posteriores. As imagens foram redimensionadas de forma que a largura e comprimento fossem iguais, tanto da máscara quanto da imagem contendo a lesão. Após vários testes, tanto de performance quanto de desempenho foi definido o tamanho $384 \times 384$, um tamanho para o qual é possível redimensionar todas as imagens, atingindo uma melhor eficiência de processamento.

Uma das principais razões para aumento de dados é a quantidade de imagens que uma rede neural necessita para ser treinada, isso foi particularmente necessário na base $\mathrm{PH}^{2}$, que possui apenas 200 imagens. Assim como afirma [Ronneberger et al. 2015], esse processo é importante para ensinar à rede a invariância e propriedades de robustez da forma desejada quando apenas algumas amostras de treinamento estão disponíveis. Sendo necessário então invariância de deslocamento e rotação, bem como robustez a deformações e variações de valor de cinza. Deformações elásticas especialmente aleatórias das amostras de treinamento são fundamentais para treinar uma rede de segmentação com poucas imagens. Foram geradas deformações suaves usando vetores de deslocamento aleatórios em uma grade de $3 \times 3$. Os deslocamentos são amostras de uma 
distribuição gaussiana com padrão de 10 pixeis de desvio. Os deslocamentos por pixel são então calculados usando interpolação bicúbica. As camadas de abandono no final do caminho de contração realizam aumentos implícitos de dados.

\subsection{U-Net: Segmentação}

Nesse trabalho utilizamos a estrutura rede U-Net proposta em [Ronneberger et al. 2015] (Figura 4). São aplicadas duas convoluções $3 \times 3$, cada uma seguida de uma unidade linear retificada (ReLU) e uma operação de pool máximo de tamanho $2 \times 2$ com passo 2 para downsampling. O restante da estrutura é seguido por uma convolução $2 \times 2$ ("convolução ascendente") e duas convoluções $3 \times 3$, cada uma seguida por uma ReLU. Sendo finalizada com uma uma convolução $1 \times 1$.

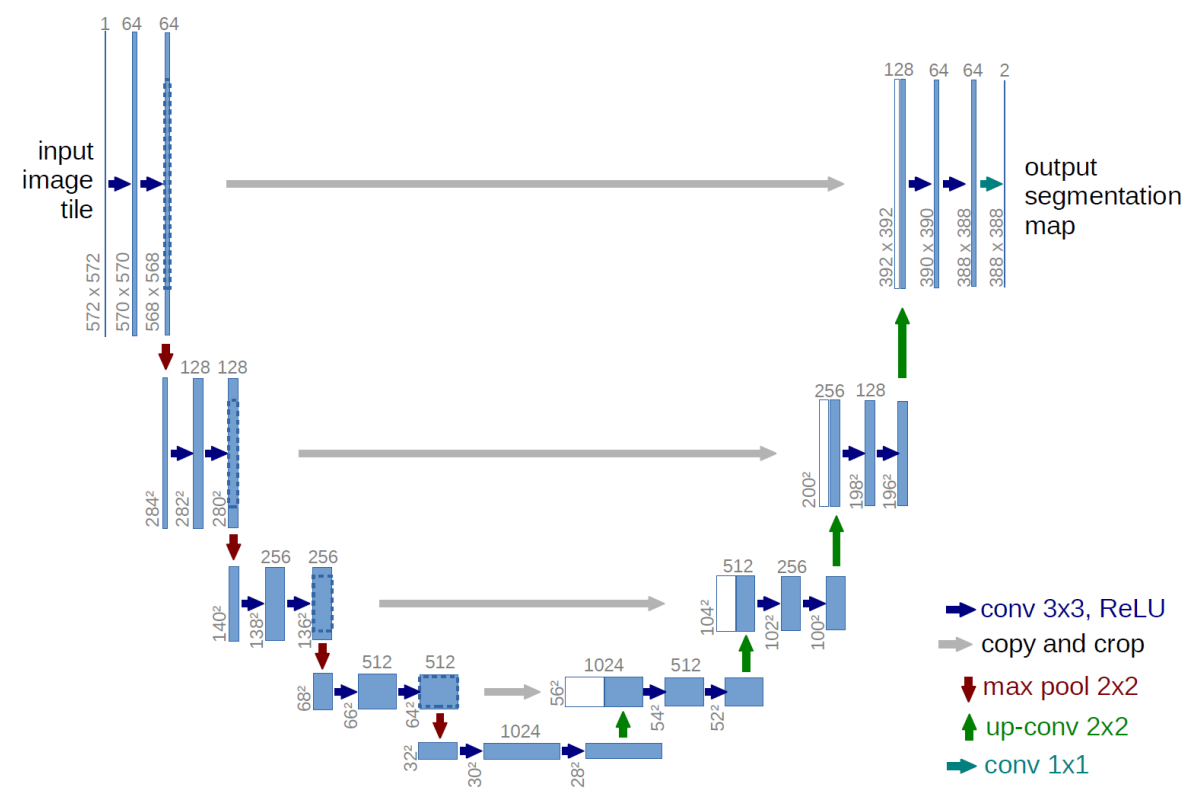

Figura 4. llustração da rede proposta por [Ronneberger et al. 2015].

Foram realizados experimentos alterando a configuração original da rede com a finalidade de obter melhores resultados. Dessa forma, foram testados parâmetros alternativos para o otimizador da rede, a quantidade de épocas e a taxa de aprendizado. Foram utilizados os otimizadores Adam e gradiente estocástico descendente (SGD) [Keras ], quando utilizado o SGD foram testadas as taxas de aprendizagem 0,0001 e 0,00001, já com Adam a taxa padrão 0,001. Além disso, foram feitos testes na rede com 200 e 1000 épocas.

\subsection{Pós-processamento}

Após a aplicação da rede U-Net utilizamos algumas técnicas de pós-processamento para melhorar os resultados obtidos. As etapas de pós-processamento foram as seguintes: 1) aplicação do limiar de Otsu; 2) fechamento de regiões; e 3) remoção de regiões pequenas.

\subsubsection{Limiar de Otsu}

As imagens resultantes da U-Net necessitam de um tratamento para que as bordas se tornem mais bem definidas. Para isso foi utilizada a técnica do limiar de Otsu, essa 
diferença pode ser notada na Figura 5, sendo de (a) a (f) imagens antes do Limiar de Otsu e de (g) a (1) após. Nota-se um contorno mais bem definido após a aplicação dessa técnica.

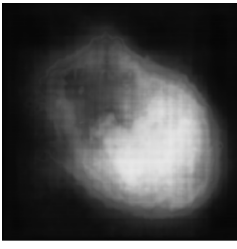

(a)

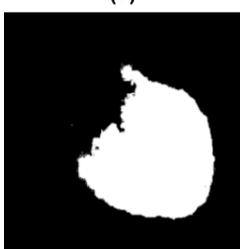

(g)

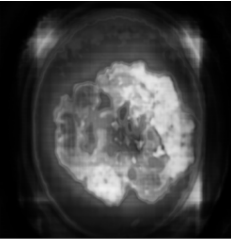

(b)

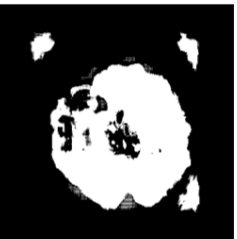

(h)

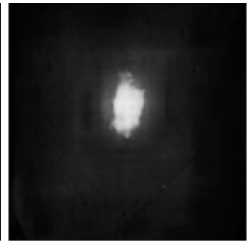

(c)

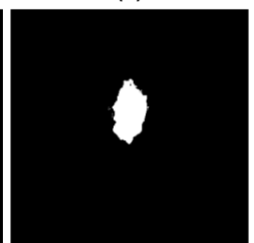

(i)

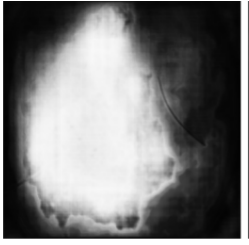

(d)

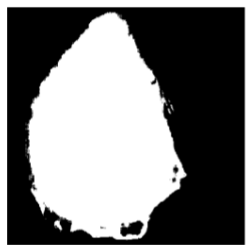

(j)

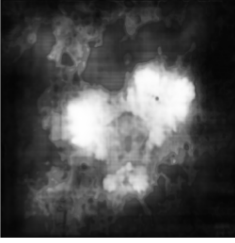

(e)

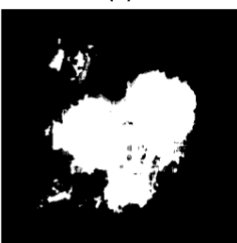

(k)

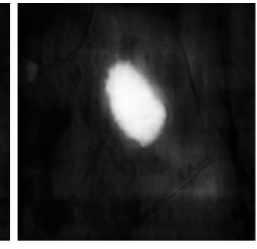

(f)

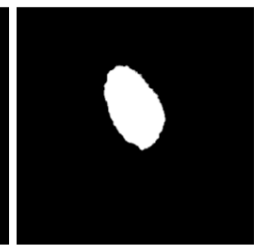

(l)

Figura 5. Imagens resultantes antes e depois da aplicação da Limiar de Otsu.

\subsubsection{Fechamento de regiões}

Após ser aplicado a limiar de Otsu percebeu-se que, em algumas imagens, haviam buracos na região, então foi aplicado o método de fechamento de regiões, é possível ver a diferença na Figura 6. O algoritmo usado nesta função consiste na invasão ou complemento das formas de entrada a partir do limite externo da imagem usando dilatações binárias. Os orifícios não estão conectados ao limite e, portanto, não são invadidos. O resultado é o subconjunto complementar da região invadida.

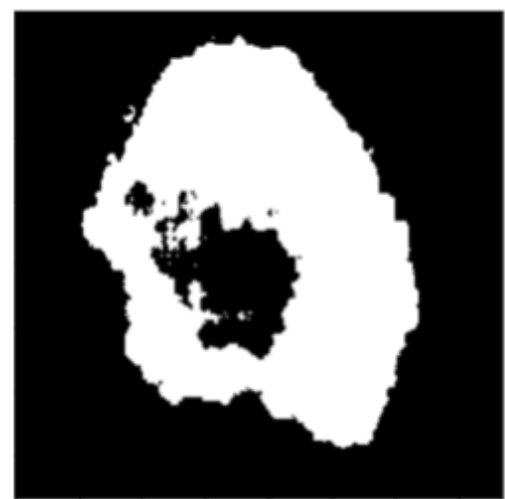

(a)

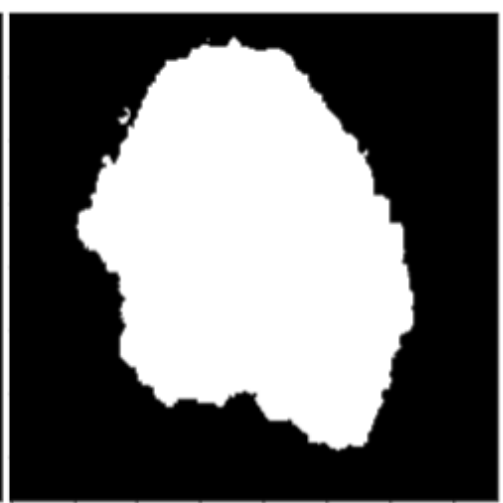

(b)

Figura 6. Exemplo de imagem com buracos (a) e após o fechamento (b).

\subsubsection{Remoção de pequenas regiões}

Em algumas imagens a rede identificou alguns elementos pequenos como lesões, sendo que a segmentação de algumas dessas regiões foram causadas por algum fator externo, 
como uma sombra na captura da imagem, por exemplo. Isso foi resolvido removendo da imagem todas as regiões com exceção da região de maior área, pois cada imagem detém apenas uma lesão de forma destacada. Na Figura 7 é possível ver como se caracteriza uma imagem contendo mais regiões além da lesão de fato.

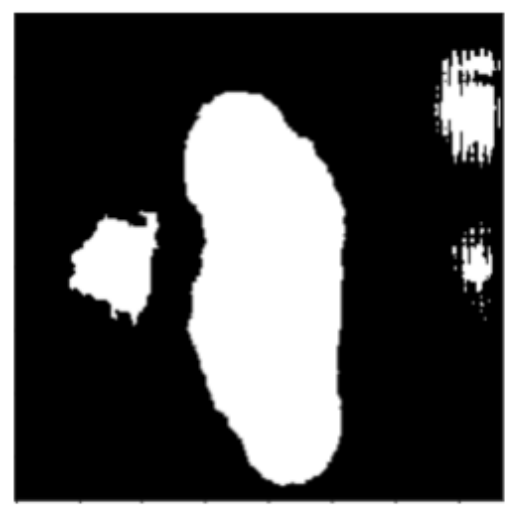

(a)

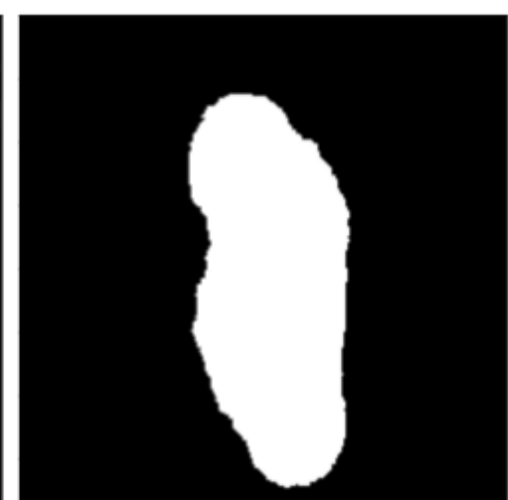

(b)

Figura 7. Exemplo de imagem com mais de uma região (a) com apenas uma região (b).

\subsection{Validação}

As métricas utilizadas para a avaliação do acerto na segmentação foram: Dice, acurácia (ACC), Jaccard (JAC), kappa (KPA) [Cohen 1968] e área sobre a curva ROC (AUC) [van Erkel and Pattynama 1998]. Para calcular essas métricas é utilizada a matriz de confusão [Provost and Kohavi 1998] na geração dos valores: Falso Negativo (FN), Falso Positivo (FP), Verdadeiro Positivo (VP) e Verdadeiro Negativo (VN). Assim as métricas são definidos como:

$$
\begin{gathered}
\text { Dice }=\frac{2,0 * T P}{(2,0 * F P)+F P+F N} . \\
A C C=\frac{V P+T P}{V N+F P+V P+F N} . \\
J A C=\frac{V P}{V P+F P+F N} .
\end{gathered}
$$

\section{Experimentos e Análise dos resultados}

Com a segmentação realizada pela rede neural U-Net, a melhor média Dice obtida com a base $\mathrm{PH}^{2}$ foi 0,4795 e com a base ISIC 2018 foi 0,3170. Após o teste de configurações de compilação na rede e com o pós-processamento foi possível obter resultados significantes, chegando a 0,9482 de Dice para a base $\mathrm{PH}^{2}$ e 0,7729 para a ISIC 2018.

Nas Tabelas 1 e 2 são mostrados todos os resultados, nas duas bases de imagens adquiridas com a mudança de configuração e em seguida com os métodos aplicados no pós-processamento. Sendo os valores providos com a métrica Dice. 
Tabela 1. Média Dice resultante da aplicação de cada etapa do pósprocessamento utilizando a base $\mathrm{PH}^{2}$.

\begin{tabular}{lllllll}
\hline Épocas & Otimizador & TA & U-Net & Otsu & PP-1 & PP-2 \\
\hline \multirow{2}{*}{200} & Adam & 0,001 & 0,4324 & 0,9132 & 0,9133 & 0,9166 \\
& SGD & 0,00010 & 0,4787 & 0,9069 & 0,9119 & 0,9129 \\
& SGD & 0,00001 & 0,4787 & 0,8345 & 0,8574 & 0,8721 \\
\hline \multirow{2}{*}{1000} & Adam & 0,001 & $\mathbf{0 , 4 7 9 5}$ & $\mathbf{0 , 9 4 6 1}$ & $\mathbf{0 , 9 4 6 5}$ & $\mathbf{0 , 9 4 8 2}$ \\
& SGD & 0,00010 & 0,4323 & 0,8855 & 0,8906 & 0,9136 \\
& SGD & 0,00001 & 0,4323 & 0,8460 & 0,8500 & 0,8878 \\
\hline
\end{tabular}

*onde TA é a taxa de aprendizado, PP-1 é o primeiro pós-processamento feito (fechamento de regiões), e PP-2 é o segundo pós-processamento feito (remoção de regiões).

Tabela 2. Média Dice resultante da aplicação de cada etapa do pósprocessamento utilizando a base ISIC 2018.

\begin{tabular}{lllllll}
\hline Épocas & Otimizador & TA & U-Net & Otsu & PP-1 & PP-2 \\
\hline \multirow{2}{*}{200} & Adam & 0,001 & 0,3167 & 0,7165 & 0,7188 & 0,7196 \\
& SGD & 0,00010 & 0,3155 & 0,6714 & 0,6742 & 0,6752 \\
& SGD & 0,00001 & 0,3155 & 0,5823 & 0,5913 & 0,6103 \\
\hline \multirow{2}{*}{1000} & Adam & 0,001 & $\mathbf{0 , 3 1 5 5}$ & $\mathbf{0 , 7 6 8 9}$ & $\mathbf{0 , 7 7 0 4}$ & $\mathbf{0 , 7 7 2 9}$ \\
& SGD & 0,00010 & 0,3170 & 0,7193 & 0,7209 & 0,7295 \\
& SGD & 0,00001 & 0,3170 & 0,6216 & 0,6320 & 0,6398 \\
\hline *onde TA é a taxa de aprendizado, PP-1 é o primeiro pós-processamento \\
feito (fechamento de regiões), e PP-2 é o segundo pós-processamento \\
feito (remoção de regiões).
\end{tabular}

Dos parâmetros testados, obtivemos melhores resultados nas duas bases de imagens utilizando o otimizador Adam e sua taxa de aprendizagem padrão $(0,001)$, e também com 1000 épocas. Podemos notar que o pós-processamento foi fundamental para o resultado final, deixando as bordas mais bem definidas com o limiar de Otsu, as imagens preenchidas com o preenchimento de regiões e a remoção de regiões provenientes de ruído com a remoção de pequenas regiões, proporcionando melhores resultados.

Para uma validação mais ampla, dispõem-se na Tabela 3 os resultados utilizando outras métricas de validação. Por ser o melhor resultado ao fim da aplicação de todos os métodos, foram utilizadas as imagens geradas com o treinamento da rede utilizando 1000 épocas para a compilação com o otimizador Adam.

Tabela 3. Resultados utilizando outras métricas com as bases PH$^{2}$ e ISIC 2018.

\begin{tabular}{lll}
\hline Métrica & PH $^{2}$ & ISIC 2018 \\
\hline Dice & 0,9482 & 0,7729 \\
ACC & 0,9678 & 0,8993 \\
JAC & 0,9041 & 0,6802 \\
KPA & 0,9152 & 0,7158 \\
AUC & 0,9581 & 0,8870 \\
\hline
\end{tabular}


Os resultados da comparação com os trabalhos presentes na literatura estão disposto nas tabelas 4 e 5 .

Tabela 4. Comparação do resultado da base $\mathbf{P H}^{2}$.

\begin{tabular}{l|l|l}
\hline Trabalho & Método & Dice \\
\hline [Bi et al. 2019] & FNC & 0,9210 \\
[Goyal et al. 2020] & FNC & 0,9070 \\
[Nazi and Abir 2020] & Otsu & 0,9300 \\
[Al-masni et al. 2018] & FrCN & 0,9138 \\
[Kim et al. 2017] & Encoder-Decoder & 0,9400 \\
Metodologia proposta & U-Net & $\mathbf{0 , 9 4 8 2}$ \\
\hline
\end{tabular}

Tabela 5. Comparação do resultado da base ISIC 2018.

\begin{tabular}{l|l|l}
\hline Trabalho & Método & Dice \\
\hline [Suk et al. 2019] & FNC & $\mathbf{0 , 8 9 8 0}$ \\
[Guo et al. 2020] & U-Net & 0,8640 \\
[Amin et al. 2020] & VGG-16 & 0,8200 \\
[Abraham and Khan 2018] & U-Net & 0,8560 \\
[Nazi and Abir 2020] & U-Net & 0,8700 \\
Metodologia proposta & U-Net & 0,7729 \\
\hline
\end{tabular}

A metodologia proposta nesse trabalho conseguiu superar os trabalhos presente na literatura quando utilizada a base de imagens $\mathrm{PH}^{2}$. Entretanto, quando utilizada a ISIC 2018 outras metodologias apresentam melhores resultados. Isso se deve ao fato que a base ISIC 2018 possuir mais imagens com condições ruins, como é o caso da Figura 8, que apresenta pelos e sombras criando mais de uma região e buracos.

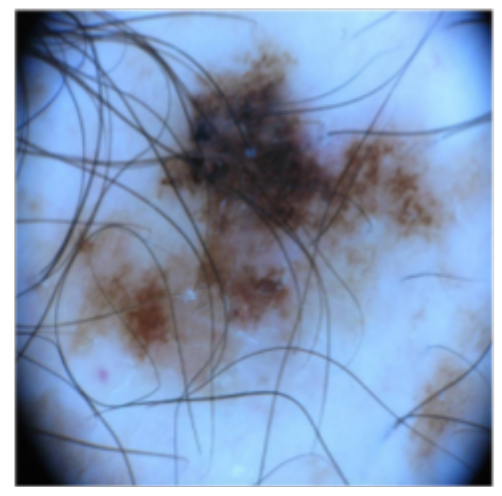

(a)

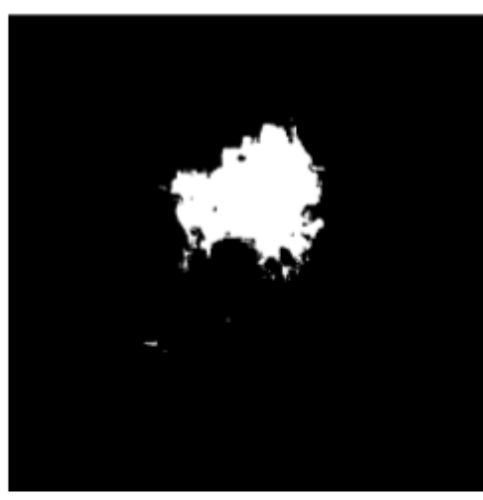

(b)

Figura 8. Exemplo de imagem com péssimas condições (a) o resultado obtido com a U-Net e aplicado a limiar de Otsu (b).

\section{Conclusões e Trabalhos futuros}

Este trabalho propõe a segmentação de imagens de lesões de câncer de pele utilizando a rede neural U-Net aliada a técnicas de pós-processamento para a otimização dos resultados obtidos. Além disso, foram testadas diferentes combinações de otimizadores da rede, número de épocas e taxa de aprendizagem da rede. 
Com base nos resultados obtidos, observa-se melhorias na base $\mathrm{PH}^{2}$ tanto quando comparada com os trabalhos relacionados quanto com os resultados na base ISIC 2018. Isso se deve, principalmente, ao fato que a ISIC 2018 apresenta mais imagens com muitos artefatos que não pertencem à lesão, tais como sombras e pelos. Desse modo, quando em melhores condições o método se provou eficiente.

Observa-se, também, uma melhoria na qualidade dos resultados decorrente da aplicação das técnicas de pós-processamento propostas às imagens resultantes da segmentação primária.

Para trabalhos futuros objetiva-se melhorar os resultados calculando mapas de textura antes da segmentação utilizando uma rede neural convolucional. Sendo os mapas: LBP, LPQ e GLCM. Além de modificar a estrutura da U-Net tradicional para resolver os problemas de desequilíbrio de dados e desempenho do treinamento, visando obter melhores resultados antes do pós-processamento. Quanto aos artefatos externos presentes na base ISIC 2018, podem ser aplicadas técnicas de pré-processamento a fim de reduzir o ruído presente nessas imagens.

\section{Referências}

[Abraham and Khan 2018] Abraham, N. and Khan, N. M. (2018). A novel focal tversky loss function with improved attention U-Net for lesion segmentation.

[Al-masni et al. 2018] Al-masni, M. A., Al-antari, M. A., Choi, M.-T., Han, S.-M., and Kim, T.-S. (2018). Skin lesion segmentation in dermoscopy images via deep full resolution convolutional networks. Computer Methods and Programs in Biomedicine, $162: 221-231$.

[Amin et al. 2020] Amin, J., Sharif, A., Gul, N., Anjum, M. A., Nisar, M. W., Azam, F., and Bukhari, S. A. C. (2020). Integrated design of deep features fusion for localization and classification of skin cancer. Pattern Recognition Letters, 131:63 - 70.

[Araújo et al. 2019] Araújo, R., e Silva, R. V., Ferreira, J., and Carvalho, N. (2019). Estudo comparativo para segmentação de melanoma em imagens de lesões de pele. In Anais da VII Escola Regional de Computação Aplicada à Saúde, pages 294-299, Porto Alegre, RS, Brasil. SBC.

[Bi et al. 2019] Bi, L., Kim, J., Ahn, E., Kumar, A., Feng, D., and Fulham, M. (2019). Step-wise integration of deep class-specific learning for dermoscopic image segmentation. Pattern Recognition, 85:78 - 89.

[Cicerone and Camp 2019] Cicerone, M. T. and Camp, C. H. (2019). 21 - potential roles for spectroscopic coherent raman imaging for histopathology and biomedicine. In Alfano, R. R. and Shi, L., editors, Neurophotonics and Biomedical Spectroscopy, Nanophotonics, pages $547-570$. Elsevier.

[Codella et al. 2019] Codella, N., Rotemberg, V., Tschandl, P., Celebi, M. E., Dusza, S., Gutman, D., Helba, B., Kalloo, A., Liopyris, K., Marchetti, M., Kittler, H., and Halpern, A. (2019). Skin lesion analysis toward melanoma detection 2018: A challenge hosted by the international skin imaging collaboration (ISIC).

[Cohen 1968] Cohen, J. (1968). Weighted kappa: nominal scale agreement provision for scaled disagreement or partial credit. Psychological bulletin, 70(4):213.

[Goyal et al. 2020] Goyal, M., Oakley, A., Bansal, P., Dancey, D., and Yap, M. H. (2020). Skin lesion segmentation in dermoscopic images with ensemble deep learning methods. IEEE Access, 8:4171-4181. 
[Guo et al. 2020] Guo, X., Chen, Z., and Yuan, Y. (2020). Complementary network with adaptive receptive fields for melanoma segmentation.

[IEEE] IEEE. $12^{\circ}$ simpósio internacional ieee de 2015 em imagem biomédica (ISBI). Disponível em https://ieeexplore.ieee.org/xpl/conhome/ 7150573 /proceeding. Acesso em 30 de março de 2020.

[Inca a] Inca. Atlas on-line de mortalidade. Disponível em https: / /www. inca . gov.br/app/mortalidade. Acesso em 28 de junho 2020.

[Inca b] Inca. Câncer de pele melanoma. Disponível em https://www. inca. gov.br/tipos-de-cancer/cancer-de-pele-melanoma. Acesso em 10 de março de 2020.

[International Skin Imaging Collaboration] International Skin Imaging Collaboration. ISIC 2018: Skin lesion analysis towards melanoma detection. Disponível em https: // challenge2018. isic-archive.com/. Acesso em 10 de março de 2020.

[ISIC] ISIC. Welcome to ISIC. Disponível em https://www.isic-archive. $\mathrm{com} / \#$ ! /topWithHeader/wideContentTop/main. Acesso em 30 de março de 2020 .

[Keras] Keras. Usage of optimizers. Disponível em https://keras.io/ optimizers/. Acesso em 12 de abril de 2020.

[Kim et al. 2017] Kim, J. U., Kim, H. G., and Ro, Y. M. (2017). Iterative deep convolutional encoder-decoder network for medical image segmentation. In 2017 39th Annual International Conference of the IEEE Engineering in Medicine and Biology Society $(E M B C)$, pages 685-688.

[Lin et al. 2020] Lin, T., Goyal, P., Girshick, R., He, K., and Dollár, P. (2020). Focal loss for dense object detection. IEEE Transactions on Pattern Analysis and Machine Intelligence, 42(2):318-327.

[Long et al. 2015] Long, J., Shelhamer, E., and Darrell, T. (2015). Fully convolutional networks for semantic segmentation. In Proceedings of the IEEE conference on computer vision and pattern recognition, pages 3431-3440.

[Mendonça et al. 2013] Mendonça, T., Ferreira, P., Marques, J., Marçal, A., and Rozeira, J. (2013). Ph2 - a dermoscopic image database for research and benchmarking. Conference proceedings:2013 35th Annual International Conference of the IEEE Engineering in Medicine and Biology Society. IEEE Engineering in Medicine and Biology Society. Conference, 2013:5437-5440.

[Ministério da Saúde] Ministério da Saúde. Câncer de pele: o que é, causas, sintomas, tratamento e prevenção. Disponível em https://saude.gov.br/ saude-de-a-z/cancer-de-pele. Acesso em 10 de março de 2020.

[Nazi and Abir 2020] Nazi, Z. A. and Abir, T. A. (2020). Automatic skin lesion segmentation and melanoma detection: Transfer learning approach with U-Net and denn-svm. In Uddin, M. S. and Bansal, J. C., editors, Proceedings of International Joint Conference on Computational Intelligence, pages 371-381, Singapore. Springer Singapore.

[Provost and Kohavi 1998] Provost, F. and Kohavi, R. (1998). Glossary of terms. Journal of Machine Learning, 30(2-3):271-274.

[Ronneberger et al. 2015] Ronneberger, O., Fischer, P., and Brox, T. (2015). U-Net: Convolutional networks for biomedical image segmentation.

[Staal et al. 2004] Staal, J., Abramoff, M. D., Niemeijer, M., Viergever, M. A., and van Ginneken, B. (2004). Ridge-based vessel segmentation in color images of the retina. IEEE Transactions on Medical Imaging, 23(4):501-509. 
[Suk et al. 2019] Suk, H.-I., Liu, M., Yan, P., and Lian, C. (2019). Machine Learning in Medical Imaging: 10th International Workshop, MLMI 2019, Held in Conjunction with MICCAI 2019, Shenzhen, China, October 13, 2019, Proceedings, volume 11861. Springer Nature.

[Universidade do Porto ] Universidade do Porto. Ph2 database. Disponível em https: //www.fc.up.pt/addi/ph2\%20database.html. Acesso em 10 de março de 2020.

[van Erkel and Pattynama 1998] van Erkel, A. R. and Pattynama, P. M. (1998). Receiver operating characteristic (roc) analysis: Basic principles and applications in radiology. European Journal of Radiology, 27(2):88 - 94.

[Zhang et al. 2020] Zhang, N., Cai, Y.-X., Wang, Y.-Y., Tian, Y.-T., Wang, X.-L., and Badami, B. (2020). Skin cancer diagnosis based on optimized convolutional neural network. Artificial Intelligence in Medicine, 102:101756. 